\title{
Development and evaluation of interdisciplinary preoperative patient education in foot and ankle surgery: immediate effects on knowledge, satisfaction and anxiety
}

\section{Entwicklung und erste Evaluation eines präoperativen interdisziplinären Schulungskonzeptes für Patienten/-innen der Fußchirurgie: unmittelbare Effekte auf Wissenszuwachs, Zufriedenheit und Angst}

\author{
Axel Schäfer ${ }^{1}$, Katrin Jettkowski², Julia Kretschmann², \\ Marco Wurg' ${ }^{2}$, Christina Stukenborg-Colsmann ${ }^{3}$, \\ Christian Plaaß ${ }^{3}$

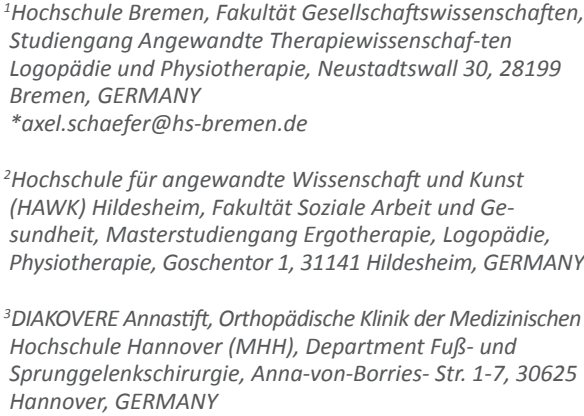

Received 10 July 2015, accepted 21 September 2016, available online 16 February 2017

\begin{abstract}
Background and objective: Foot and ankle surgery is increasing due to demographic and lifestyle changes. Most often, pa-tients are required to unload their foot postoperatively, resulting in significant impairment of activi-ties of daily living without adequate preparation for this situation. The aim of the study was the development and evaluation of a preoperative patient education intervention.

Methods: Based on current research, a patient education intervention was developed. To investigate immediate effects, a longitudinal study with two points of measurement before and after the education session was conducted. Outcomes were increase in knowledge, satisfaction and preoperative anxiety, which were measured with a selfdeveloped questionnaire. Additionally, five short interviews were conducted.

Results: 56 patients $(63 \%$ female) with a mean age of $56,7(S D=14,8)$ years were included. Patients had a significant increase in knowledge following the intervention from mean $3,93(S D=1,78)$ to $5,48(S D=2,29)$ correctly answered questions (out of 10$)$. Patients felt that they were better informed and more satisfied with the information provided. Patients evaluated the way of information delivery, the content of information, the group and the location as positive. The expectations of patients relating to postoperative loadbearing capacity and coping with eve-ryday life as well as preoperative anxiety did not change following the intervention.

Conclusion: The education intervention could be implemented in the clinical routine and showed positive ef-fects. However regarding realistic expectations and preoperative anxiety no change was ob-served. Long-term post operative effectivity and efficiency of patient education should be further examined within a randomized controlled trial.
\end{abstract}

\footnotetext{
Abstract

Hintergrund und Ziel: Chirurgische Eingriffe an Fuß- und Sprunggelenk nehmen aufgrund des demographischen Wandels und den sich ändernden Lebensgewohnheiten kontinuierlich zu. Meist besteht postoperativ die Indikation zur Entlastung, wodurch Patienten/-innen mit erheblichen Einschränkungen im Alltag konfrontiert werden, ohne darauf adäquat vorbereitet zu sein. Das Ziel der Studie war daher die Entwicklung und Evaluation einer präope-rativen Patienten/-innenschulung.

Methode: Auf Basis aktueller Forschungsergebnissen wurde eine interdisziplinäre Schulungsmaßnahme entwickelt. Eine Längsschnittstudie mit zwei Messzeitpunkten wurde durchgeführt, um die Effektivität der Schulung für die Endpunkte Wissenszuwachs, Zufriedenheit sowie präoperative Angst zu untersuchen. Zusätzlich wurden fünf Kurzinterviews durchgeführt. Ergebnisse: 56 Patienten/-innen (63\% Frauen) mit einem mittleren Alter von 56,7 Jahren $(s=14,8)$ Jahren nahmen teil. Es zeigte sich ein Wissenszuwachs von 3,93 $(s=1,78)$ auf $5,48(s=2,29)$ richtig beantworteten Fragen (von 10) nach der Schulung $(p<$ $0,001)$. Patienten/-innen fühlten sich nach der Veranstaltung besser informiert und aufgeklärt $(p<0,001)$. Auch die Zufriedenheit mit den Informationen und der Betreuung war nach Schulung besser $(p<0,001)$. Die Art der Infor-mationsvermittlung, Ablauf der Schulung, Teilnehmer/-innengruppe, Räumlichkeiten, Informati-onsgehalt sowie der Gesamteindruck wurde von den Teilnehmenden
} 
als positiv bewertet. Die Erwartungen der Patienten in Hinblick auf Belastbarkeit und Alltagsbewältigung sowie die präoperative Angst veränderten sich nicht.

Schlussfolgerung: Die Schulungsmaßnahme ließ sich gut in den Klinikablauf implementieren und zeigte positive Effekte. Im Hinblick auf eine realistischere Einschätzung der postoperativen Situation sowie der präoperativen Angst zeigte sich allerdings kein Einfluss. Die langfristige Wirksamkeit der Schu-lungsmaßnahme auf Angst, Zufriedenheit und funktionelles Ergebnis sowie die Effizienz sollte weiterführend im Rahmen einer kontrollierten Studie untersucht werden.

\section{Keywords}

Preoperative patient education - foot and ankle surgery - health literacy - satisfaction - anxiety

\section{Keywords}

Präoperative Patientenschulung - Patientenedukation - Fußchirurgie - Sprunggelenkschirurgie - Zufriedenheit - Angst

\section{EINLEITUNG}

Die Anzahl chirurgischer Eingriffe am Fuß (Mittelfußknochen und Zehen) ist von 96.433 (Stand 2005) auf 144.287 (Stand 2011) angestiegen, dies entspricht einer Steigerung um ca. $66 \%$ (GBE des Bundes, 2012). Bei einem Großteil ist postoperativ eine Entlastung der betroffenen Extremität von sechs bis acht Wochen empfohlen (Barg et al., 2011; Eyfferth et al., 2011; Niggermeyer et al., 2011; Ruffing et al., 2011; Zwipp \& Grass, 2005). Dies führt postoperativ zu gravierenden Einschränkungen der Selbständigkeit im Alltag. Die meisten Patienten/-innen unterschätzen diese Beeinträchtigungen, wodurch es zu unrealistischen Erwartungshaltungen und einer negativen Auswirkung auf die postoperative Zufriedenheit kommen kann (Heikkinen et al., 2007).

Fußoperierte Patienten/-innen sind laut Bundesarbeitsgemeinschaft für Rehabilitation (BAR, 2005) nicht rehabilitationsfähig. Ihnen steht somit auch keine stationäre Anschlussheilbehandlung zu. Aus diesem Grund ist es notwendig, Patienten/-innen optimal auf die Zeit nach der Entlassung vorzubereiten. Eine Möglichkeit besteht darin, präoperative Schulungen durchzuführen, um relevante Informationen zur postoperativen Situation zu vermitteln.

Verschiedene Studien zeigten, dass präoperative Patienten/innenschulungen bei Hüft- und Knieoperationen die Angst vor der Operation (OP) reduzieren (Soever et al., 2010; Bondy et al., 1999; Giraudet-Le Quintrec et al., 2003; Prouty et al. 2006) und die Zufriedenheit nach der OP erhöhen können (Heikinen et al., 2007; Johansson et al., 2007). Die Zufriedenheit nach der OP ist dabei von präoperativ realistischen Erwartungen abhängig (McGregor et al., 2004; Bonnin et al., 2011). Eine präoperative Patienten/-innenschulung kann außerdem die Fähigkeit zur selbständigen Durchführung der Aktivitäten des täglichen Lebens (ADL) nach der OP positiv beeinflussen (Coudeyre et al., 2007). Eine Metaanalyse von McDonald et al. (2008) weist allerdings auf heterogene Ergebnisse bezüglich Angst, Zufriedenheit und ADL hin.
Bisher wurde der Nutzen von Patienten/-innenschulungen bei Eingriffen an Sprunggelenk und Fuß nur in einer Studie aus England untersucht (Selvan et al., 2013). In dieser Untersuchung wurden positive Effekte einer präoperativen Schulung in Bezug auf die Dauer des Krankenhausaufenthaltes und die Patienten/innenversorgung nachgewiesen.

In der Literatur finden sich Empfehlungen in Bezug auf Inhalt, Form und Durchführung von präoperativen Schulungsmaßnahmen. Schulungskonzepte stellten sich dann als besonders wirksam dar, wenn sie von multidisziplinären Teams in Kleingruppen durchgeführt wurden (Soever et al., 2010; Giraudet-Le Quintrec et al., 2003). Auch die Bedeutung von praktischen Übungen innerhalb einer Schulung zeigte sich in den signifikanten Verbesserungen der postoperativen ADL-Fähigkeiten (Coudeyre et al., 2007; Fortin \& Kirouac, 1976). Effizient scheint eine Kombination aus praktischen Übungen und theoretisch vermittelten Informationen zu sein. Auch der Einsatz verschiedener Medien sowie die Ausgabe von schriftlichem Informationsmaterial zeigten positive Einflüsse auf die Erwartungshaltungen und die postoperative Zufriedenheit (Johnson et al., 2008; Kaymakcı et al., 2007; McGregor et al., 2004; Cooil \& Bithell, 1997).

Für den deutschsprachigen Raum gibt es bisher noch keine Studien, die die Wirksamkeit von Patienten/innenschulungen im Zusammenhang mit fußchirurgischen Eingriffen untersuchten. Das Ziel der vorliegenden Arbeit ist daher die Entwicklung und erste Evaluation einer präoperativen Schulung für Patienten/-innen mit Fußund Sprunggelenksproblemen. Die Schulung soll die Informiertheit der Patienten/-innen in Bezug auf die geplante fußchirurgische OP, die Nachbehandlung und die damit in Verbindung stehenden Themen nachhaltig verbessern. Die leitende Forschungsfrage lautet: Kann eine präoperative Schulung bei Patienten mit geplantem fußchirurgischem Eingriff (mit postoperativer Entlastung oder Teilbelastung) unmittelbar den Wissensstand erhöhen und den subjektiven Zugewinn (z. B. Zufriedenheit mit der Aufklärung, weniger Angst vor der OP, realistische Erwartungen in Bezug auf Funktion und ADL nach der OP) beeinflussen? 


\section{METHODE}

\section{Konzeption der Schulung}

Das Schulungskonzept basiert auf einem interdisziplinären Ansatz und vereint drei Aspekte präoperativer Schulungskonzepte: Kleingruppen, Medienvielfalt und praktisches Üben (Johnson et al., 2008, vgl. Abbildung 1).

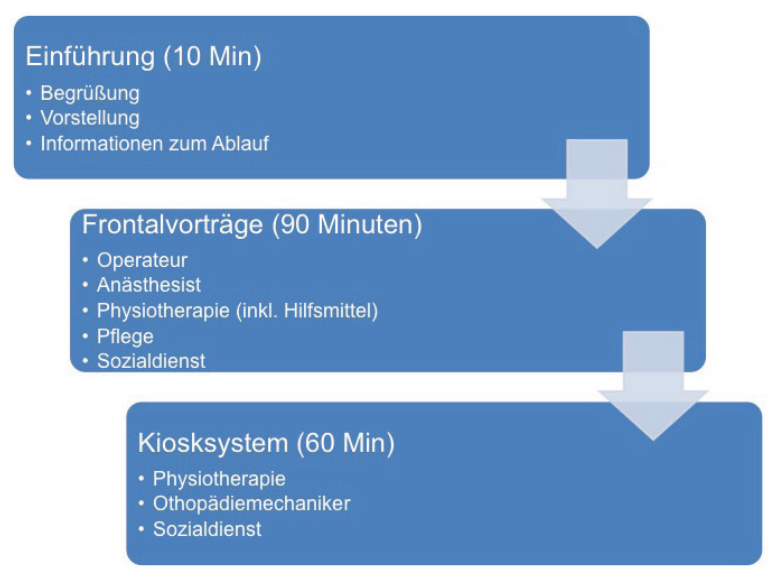

Abbildung 1: Ablauf der Schulung

Das Schulungsteam bestand aus Fachkräften, die die Patienten/-innen mit Vorträgen allgemein über fußchirurgische Eingriffe, Anästhesie, die pflegerische, sozialdienstliche und physiotherapeutische Versorgung im Krankenhaus, die postoperative Nachbehandlungsphase und die Versorgung mit Hilfsmitteln aufklärten. Im Anschluss an die Information konnten die Patienten/-innen sichanInformationsständeninformierenundan praktischen Übungen zum entlasteten Gehen mit Hilfsmitteln teilnehmen. Nach der Schulung und Evaluation erhielten sie eine Broschüre mit allen relevanten Schulungsinhalten, Übungen und Kontaktadressen. Für die Intervention stand ein großer Saal in der orthopädischen Klinik der Medizinischen Hochschule Hannover im Annastift zur Verfügung. Die 2,5 Stunden umfassende Schulung wurde von einem erfahrenen und geschulten Team des Fuß- und Sprunggelenkdepartments des Annastifts (Krankenhaus in Hannover) bestehend aus Chirurgen/innen, Physiotherapeuten/-innen, Stationspflege, Anästhesie, Mitarbeitern/-innen des Sozialdienstes und einem Orthopädietechniker durchgeführt.

\section{Studiendesign und Ablauf}

Zur Darstellung unmittelbarer Effekte nach der Schulung wurde das Design einer Längsschnittstudie mit zwei Messzeitpunkten am Schulungstag gewählt. Vor Beginn der Maßnahme (t0) erhielten die Patienten/-innen eine schriftliche Information über Ziele und Ablauf der Studie, eine schriftliche Einverständniserklärung sowie den zeitlichen Ablaufplan der Schulung und die Fragebögen. Die zweite Messung (t1) erfolgte unmittelbar nach der Schulung. Es wurden insgesamt drei Schulungen durchgeführt.

\section{Probanden: Ein- und Ausschlusskriterien}

Einschlusskriterium für die Studie war eine geplante fußchirurgische Operationen mit Notwendigkeit zur postoperativen Entlastung. Es gab keine Altersbeschränkungen. Ausgeschlossen wurden Patienten/-innen mit Revisionen und akuten fußchirurgischen Eingriffen. Die Rekrutierung erfolgte anhand der OP-Warteliste des Annastiftes. Entsprechend der Ein- und Ausschlusskriterien wurden insgesamt 249 Patienten/-innen eingeladen. Davon nahmen 63 an der Schulung teil.

\section{Endpunkte}

Endpunkte der Studie waren der Wissenszuwachs, der subjektiv empfundene Zugewinn und die Zufriedenheit mit der Schulung.

\section{Datenerhebung}

Die Datenerhebung erfolgte mit einem spezifisch für die Schulungsmaßnahme entwickelten dreiteiligen Evaluationsinstrument (Jettkowski et al., 2013). Die Verständlichkeit und interne Konsistenz der Fragebögen wurde vor Einsatz in der Studie im Rahmen eines Pretests untersucht und der Fragebogen entsprechend angepasst.

\section{Skala Wissenszuwachs}

Die Skala Wissenszuwachs besteht aus 10 MultipleChoice-Fragen, die sich auf das Wissen beziehen, das den Patienten/-innen in der Schulung vermittelt wurde. Dies beinhaltet das richtige Verhalten vor und nach der Operation, wie etwa die Notwendigkeit der Entlastung des operierten Fußes, Verbandswechsel und Maßnahmen bei Schwellung und Schmerzen. Pro richtig beantwortete Frage wird ein Punkt vergeben, das theoretische Minimum liegt entsprechend bei 0 Punkten, das theoretisches Maximum bei 10 Punkten. Die Fragen wurden zu den Messzeitpunkten t0 und t 1 erhoben.

\section{Subskalen zu subjektivem Zugewinn}

Der subjektive Zugewinn wurde anhand von sieben Subskalen zu den Zeitpunkten t0 und t1 gemessen. Die 


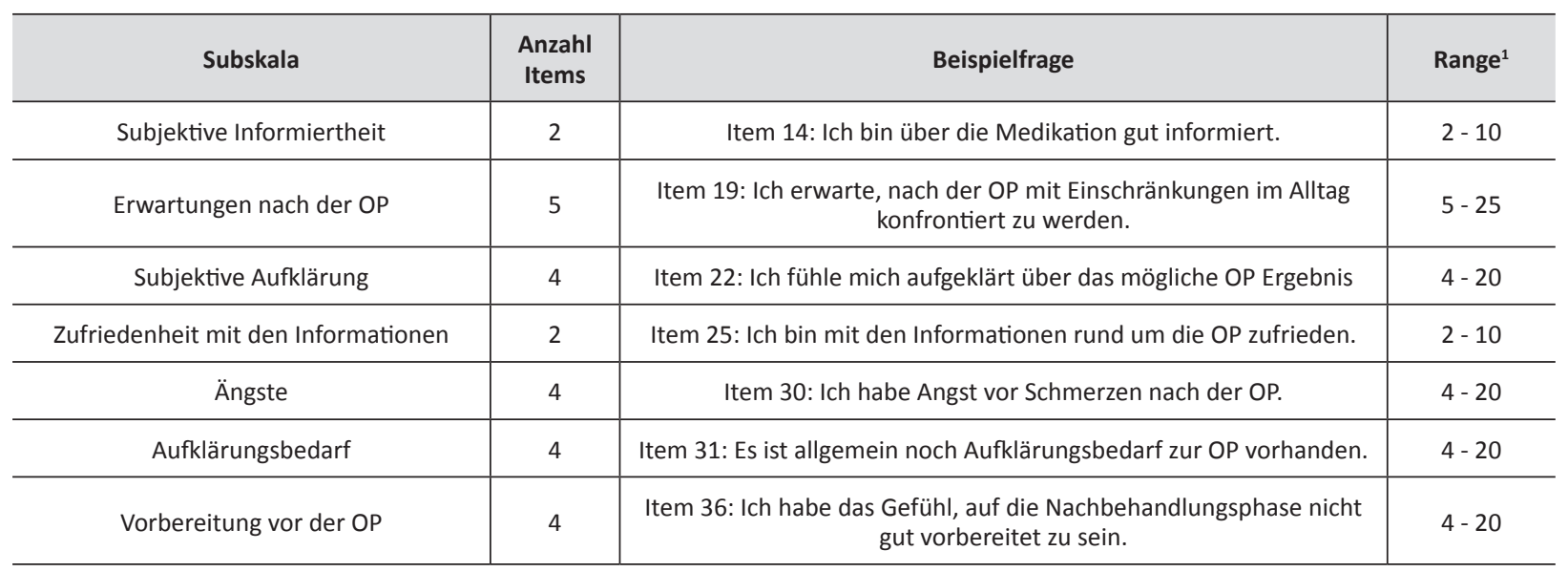

${ }^{1}$ Die Einzelitems werden mittels einer 5-stufigen Likert Skala (1 = trifft voll zu - 5 = trifft gar nicht zu) beantwortet. Die Skalen wurden so recodiert, dass hohe Punktzahlen einer hohen Ausprägung der jeweiligen Dimension entsprechen

Skala umfasst 25 Items zu subjektiver Zufriedenheit in Bezug auf die vermittelten Informationen, zu Erwartungen, präoperativer Aufklärung, der Zufriedenheit mit den Informationen, Ängsten, dem Aufklärungsbedarf und der Vorbereitung auf die Zeit nach der OP (Tabelle 1). Das Antwortformat reichte jeweils von $1=$ trifft voll zu bis 5 $=$ trifft gar nicht $\mathrm{zu}$.

\section{Subskalen zur Schulungsevaluation}

Die Zufriedenheit mit dem Schulungskonzept wurde mit vier Subskalen bestehend aus 24 Items und zwei Einzelitems unmittelbar nach der Schulung erhoben (t1). Diese beziehen sich auf die Zufriedenheit mit der Art der Informationsvermittlung, dem Ablauf der Schulung, der Teilnehmer/-innengruppe, den Räumlichkeiten, dem Informationsgehalt und dem Gesamteindruck. Das Antwortformat reichte jeweils von $1=$ trifft voll zu bis 5 $=$ trifft gar nicht zu (Tabelle 2).

\section{Kurzinterviews}

Begleitend zur quantitativen Erhebung wurden mit dem Schulungsteam und zufällig ausgewählten Patienten/innen kurze leitliniengestützte Interviews von 5-10 Minuten Dauer durchgeführt. Hierbei wurden Aspekte zu Rahmenbedingungen, Ablauf, zu den Teilnehmenden und dem Schulungsteam erhoben, um ein direktes und offenes Feedback einzuholen. Die Gespräche wurden handschriftlich protokolliert.

\section{Methoden der Datenanalyse}

Es wurden deskriptive Verfahren zur Beschreibung der Stichprobe verwendet. Unterschiedshypothesen wurden mit dem t-Test für abhängige Stichproben getestet. Die Skalen wurden so recodiert, dass hohe Punktezahlen eine starke Ausprägung der jeweiligen Dimension anzeigen. Die statistische Auswertung der erhobenen Daten wurde mit dem Statistikprogramm IBM SPSS Statistics 21 für Windows durchgeführt. Das Signifikanzniveau wurde bei $\alpha=0,05$ festgelegt. Die qualitativen Daten aus den Kurzinterviews wurden inhaltsanalytisch ausgewertet.

\section{Ethik}

Die ethische Unbedenklichkeit der Studie wurde von der Ethikkommission der Medizinischen Hochschule Hannover bescheinigt (Ethikvotum Nr. 1418-2012).

\section{ERGEBNISSE}

Im Folgenden werden die Ergebnisse der quantitativen und qualitativen Datenanalyse dargestellt.

\section{Stichprobenbeschreibung}

Insgesamt 63 Personen nahmen an den drei Schulungen teil. Von 56 Personen lagen Fragebögen und schriftliche Einverständniserklärungen vor, davon waren $63 \%$ Frauen. Das mittlere Alter betrug 56,7 Jahre $(\mathrm{s}=14,8)$. Bei $24 \%$ der Probanden/-innen wurden bisher noch keine Operationen durchgeführt. $58 \%$ der Befragten gaben an, die beste Aufklärung bezüglich der Operation bisher durch den Operateur erhalten zu haben. Die Mehrzahl der Teilnehmenden erwarteten vor der Schulung, nach vier $(22,6 \%)$, sechs $(32,1 \%)$ bzw. zwölf $(35,8 \%)$ Wochen nach der Operation die Alltagsaktivitäten ohne Einschränkung wieder aufnehmen zu können, nur eine Minderheit erwartete dies unmittelbar nach dem 
Tabelle 2: Subskalen zur Zufriedenheit mit dem Schulungskonzept

\begin{tabular}{c|c|c|c}
\hline Subskala & Anzahl Items & Beispielfrage & Range $^{\mathbf{1}}$ \\
\hline Art der Informationsvermittlung & 6 & Item 40: Die Vorträge waren allgemein gut verständlich & 6 - 30 \\
\hline Ablauf der Schulung & 9 & Item 53: Die Zeit um Fragen zu stellen war ausreichend & $9-45$ \\
\hline Teilnehmer/innengruppe & 4 & Item 54: Die Teilnehmer/innenanzahl der Gruppe war zu groß & $4-20$ \\
\hline Räumlichkeiten & 5 & $\begin{array}{c}\text { Item 60: Die Räumlichkeiten bieten genügend Platz für die } \\
\text { Umsetzung der praktischen Übungen }\end{array}$ & 5 - 25 \\
\hline
\end{tabular}

${ }^{1}$ Die Einzelitems werden mittels einer 5-stufigen Likert Skala $(1=$ trifft voll zu - 5 = trifft gar nicht zu) beantwortet. Die Skalen wurden so recodiert, das hohe Punktzahlen einer hohen Ausprägung der jeweiligen Dimension entsprechen

Krankenhausaufenthalt $(1,9 \%)$ bzw. eine Woche danach (7,5\%). Der Zeitraum vom Schulungszeitpunkt bis zum Operationstermin betrug zwischen 3 und 15 Wochen.

\section{Wissenszuwachs}

Zehn Fragen zielten auf das Wissen der Patienten/-innen in Bezug auf das richtige Verhalten vor und nach der Operation ab, die Teilnehmenden konnten also maximal zehn richtige Antworten geben. Geprüft wurde, ob durch die Schulung ein Wissenszuwachs verzeichnet werden konnte. Die Patienten verbesserten sich im Mittel von 3,93 $(\mathrm{s}=1,78)$ richtig beantwortete Fragen vor der Schulung auf 5,48 $(\mathrm{s}=2,29)$ richtig beantwortete Fragen nach der Schulung mit $\mathrm{t}_{55}=-4,40 ; \mathrm{p}=0,000$.

\section{Subjektiver Zugewinn}

Signifikant positive Effekte der Schulung konnten für einzelne Subskalen nachgewiesen werden (Tabelle 4). Patienten/-innen fühlten sich nach der Schulung besser informiert $(\mathrm{p}=0,000)$ und aufgeklärt $(\mathrm{p}=0,000)$. Die Teilnehmenden waren zudem zufriedener mit den Informationen rund um die Operation und dem Behandlungsverlauf $(p=0,000)$ und hatten nach der Schulung weniger Bedarf an Aufklärung $(\mathrm{p}=0,000)$. Patienten/-innen fühlten sich besser vorbereitet auf die OP $(p=0,006)$. Keinen Einfluss hatte die Schulung auf die Parameter präoperative Angst $(\mathrm{p}=0,954)$ und Erwartungen an die Situation nach der Operation $(\mathrm{p}=0,258)$.

\section{Ergebnisse der Schulungsevaluation}

Die Teilnehmenden bewerteten die Art der Informationsvermittlung, den organisatorischen Ablauf, die Teilnehmendenzahl, die Räumlichkeiten und die Relevanz der behandelten Themen als angemessen (Tabelle 5).
Tabelle 3: Beschreibung der Stichprobe

\begin{tabular}{|c|c|c|c|}
\hline & & Häufigkeit & $\begin{array}{l}\text { Gültige } \\
\text { Prozente }\end{array}$ \\
\hline Alter MW (s) & $56,7(14,8)$ & & \\
\hline \multicolumn{4}{|l|}{ Geschlecht } \\
\hline \multirow[t]{3}{*}{ Gültig } & $\mathrm{m}$ & 18 & 37,3 \\
\hline & w & 32 & 62,7 \\
\hline & Gesamt & 50 & \\
\hline Fehlend & & 6 & \\
\hline Gesamt & & 56 & 100 \\
\hline \multicolumn{4}{|c|}{ Bisher durchgeführte $O P$} \\
\hline \multirow[t]{6}{*}{ Gültig } & 0 & 13 & 24,1 \\
\hline & 1 & 7 & 13,0 \\
\hline & 2 & 11 & 20,4 \\
\hline & 3 & 6 & 11,1 \\
\hline & $>3$ & 17 & 31,5 \\
\hline & Gesamt & 54 & \\
\hline Fehlend & & 2 & \\
\hline Gesamt & & 56 & 100 \\
\hline \multicolumn{4}{|c|}{ Beste Aufklärung durch } \\
\hline \multirow[t]{7}{*}{ Gültig } & Internet & 1 & 2,3 \\
\hline & Bekannte & 1 & 2,3 \\
\hline & Hausarzt/-ärztin & 10 & 23,3 \\
\hline & Operateur/-in & 25 & 58,1 \\
\hline & vorherige OP & 4 & 9,3 \\
\hline & spezielle Schulung & 2 & 4,7 \\
\hline & Gesamt & 43 & 100 \\
\hline Fehlend & & 13 & \\
\hline Gesamt & & 56 & \\
\hline
\end{tabular}

$M W=$ Mittelwert,$s=$ Standardabweichung, $O P=$ Operation

\section{Ergebnisse der Kurzinterviews}

Die Auswertung der fünf Kurzinterviews zeigte, dass sowohl das Schulungsteam als auch die Teilnehmenden allgemein mit dem Ablauf der Schulung zufrieden 
Tabelle 4: Ergebnisse zu den Subskalen subjektiver Zugewinn

\begin{tabular}{|c|c|c|c|c|c|c|}
\hline \multirow[t]{2}{*}{ Subskala } & \multicolumn{2}{|c|}{ to } & \multicolumn{2}{|c|}{ t1 } & \multirow[b]{2}{*}{$t^{1}$} & \multirow[b]{2}{*}{$\mathbf{p}$} \\
\hline & MW & s & MW & s & & \\
\hline Subjektive Informiertheit & 4,86 & 2,76 & 9,11 & 1,18 & $-8,58$ & ,000 \\
\hline Erwartungen nach der OP & 7,79 & 1,97 & 8,43 & 2,94 & $-1,15$ & ,258 \\
\hline Subjektive Aufklärung & 12,75 & 4,47 & 17,86 & 2,29 & $-6,06$ & ,000 \\
\hline Zufriedenheit & 6,37 & 2,37 & 8,86 & 1,26 & $-6,08$ & ,000 \\
\hline Ängste & 11,26 & 4,16 & 11,29 & 4,79 & $-0,06$ & ,954 \\
\hline Weiterer Aufklärungsbedarf & 13,42 & 5,14 & 8,00 & 2,49 & 5,58 & , 000 \\
\hline Vorbereitung auf die OP & 12,74 & 2,93 & 14,15 & 3,46 & $-2,91$ & ,006 \\
\hline
\end{tabular}

${ }^{1}$ t-Test für abhängige Stichproben.

$M W=$ Mittelwert; $s=$ Standardabweichung

Die Einzelitems werden mittels einer 5-stufigen Likert Skala (1 = trifft voll zu - 5 = trifft gar nicht zu) beantwortet. Die Skalen wurden so recodiert, dass hohe Punktzahlen einer hohen Ausprägung der jeweiligen Dimension entsprechen

Tabelle 5: Ergebnisse zu den Subskalen der Schulungsevaluation

\begin{tabular}{c|c|c|c|c}
\hline Subskala & \multicolumn{2}{|c}{ t1 } & MW & s \\
\hline Bewertung der... & theor. Min. & Theor. Max. & 30 & 27,59 \\
\hline Art der Informationsvermittlung & 6 & 45 & 40,33 & 4,47 \\
\hline Ablauf der Schulung & 9 & 20 & 17,44 & 2,73 \\
\hline Teilnehmer/innengruppe & 4 & 25 & 22,53 & 3,03 \\
\hline Räumlichkeiten & 5 & 5 & 4,10 & 0,98 \\
\hline Informationsgehalt (Einzelitem) & 1 & 5 & 4,54 & 0,62 \\
\hline Gesamteindruck (Einzelitem) & 1 & & \\
\hline
\end{tabular}

$M W=$ Mittelwert $s=$ Standardabweichung

Die Einzelitems werden mittels einer 5-stufigen Likert Skala (1 = trifft voll zu - 5 = trifft gar nicht zu) beantwortet. Die Skalen wurden so recodiert, dass hohe Punktzahlen einer positven Bewertung der jeweiligen Dimension entsprechen

waren. Die Möglichkeit der individuellen Beratung an den Informationsständen wurde als sehr gut bewertet und angenommen. Die heterogene Zusammensetzung der Gruppe und der daraus resultierende rege Austausch wurden positiv bewertet. Es wurden konstruktive Vorschläge $\mathrm{zu}$ organisatorischen und inhaltlichen Aspekten geäußert, die zur Optimierung des Konzeptes weiterverwendet werden können. Die Teilnehmenden fühlten sich nach der Schulung besser informiert und aufgeklärt. Zwei Teilnehmende berichteten, ihnen sei durch die Schulung bewusst geworden, dass sie mit den Einschränkungen nach der Operation zum aktuellen Zeitpunkt nicht angemessen umgehen können und sie aus diesem Grund den Operationstermin verschieben müssen. Insgesamt wurden die praktischen Übungen als sehr hilfreich empfunden. Eine Interviewpartnerin bemerkte, dass der Umgang mit Hilfsmitteln sich deutlich von dem unterschieden hat, was ihr bei einer vorangegangenen Knieoperation vermittelt worden war.

\section{DISKUSSION}

Nach einer interdisziplinären Schulungsmaßnahme verzeichneten Patienten/-innen einen signifikanten Wissenszuwachs in Bezug auf die OP-Vorbereitung und Nachsorge des operierten Fußes, auch der subjektive Grad der Informiertheit, der Aufklärungsbedarf und die Zufriedenheit mit den Informationen verbesserten sich signifikant.

\section{Wissenszuwachs}

In Bezug auf den Wissensstand konnte ein signifikanter Zuwachs nach der Schulung nachgewiesen werden. 
Kritisch betrachtet werden muss in diesem Fall, dass nicht alle Teilnehmenden jede Frage beantworteten und die Ergebnisse mit Blick auf die jeweils gültigen Antworten betrachtet werden müssen.

\section{Subjektiver Zugewinn}

In der Betrachtung des subjektiven Zugewinns erwies sich die Schulung sinnvoll, da Einflüsse auf die subjektive Informiertheit und auf die Zufriedenheit mit den vermittelten Informationen dargestellt werden konnten. Ein positiver Effekt der Maßnahme auf den Bedarf an Aufklärung und dem Gefühl vorbereitet zu sein, unterstützt den Nutzen der Schulung. Dies deckt sich mit den Ergebnissen einer aktuellen Untersuchung einer präoperativen Gruppenintervention bei fußchirurgischen Patienten/-innen, auch hier zeigten sich positive Effekte bezüglich einer besseren Vorbereitung auf die Situation nach der OP (Selvan et al., 2013).

\section{Präoperative Angst}

Ein positiver Einfluss der Schulung auf die Angst konnte in dieser Untersuchung nicht nachgewiesen werden. Ein Grund hierfür könnte der große Abstand von bis zu 15 Wochen zur OP sein. Einen Zusammenhang zwischen zeitlicher Distanz zur OP und der präoperativen Angst konnten etwa Bondy et al. (1999) nachweisen. Sie fanden heraus, dass die Angst unmittelbar vor der OP signifikant am stärksten ausgeprägt ist. Eine große Distanz zum OP-Termin ist somit tendenziell eher ungeeignet, um eine Veränderung der präoperativen Angst abbilden zu können. Für diesen Aspekt scheint es sinnvoll, noch weitere Messzeitpunkte einzubauen.

\section{Stärken und Schwächen der Studie}

Stärken dieser Beobachtungsstudie sind die sehr realitätsnahen Versuchsbedingungen und eine daraus resultierende hohe externe Validität. Die Schulung wurde unter Alltagsbedingungen durchgeführt, auch die Zusammensetzung der Stichprobe entsprach bezüglich Alter und Geschlechterverteilung der Zielpopulation (vgl. GBE des Bundes, 2012).

Das Schulungskonzept wurde auf Grundlage einer systematischen Literaturrecherche an aktuelle Forschungsergebnisse angepasst und individuell auf das Setting und die Zielgruppe zugeschnitten. Entsprechend verfügte das auf Fußchirurgie spezialisierte interdisziplinäre Schulungsteam bereits über Erfahrung in der Durchführung von Patienten/-innenschulungen.

Der kurze Erhebungszeitraum im Prä-Post-Design ermöglichte ein direktes Feedback und ein Einfangen der aktuellen Stimmung am Schulungstag. Zudem waren die Teilnehmenden direkt vor Ort und die Evaluation brauchte nicht auf dem Postweg erfolgen. Dies reduzierte die Anzahl der Dropouts und gewährleistete trotz kleiner Stichprobe einen guten Rücklauf.

Die Fragebögen wurden eigens für die Schulung und die Zielgruppe konstruiert und zeigten in einem Pretest an Patienten/-innen der Zielpopulation gute Verständlichkeit und eine hohe interne Konsistenz. Auch der Einbezug von qualitativen Interviews im Sinne einer Methodentriangulation ist eine weitere Stärke der Studie, die eine Einordnung der Ergebnisse der Fragebogenerhebung ermöglicht.

Auch wenn aus pragmatischen Gründen keine Verblindung der Datenaufnahme und -auswertung möglich war, so erfolgte diese doch unabhängig von der durchführenden Institution durch die HAWK.

Schwächen der Studie sind mögliche Verzerrungsfaktoren durch die relativ große Anzahl unvollständiger Datensätze, die fehlende Kontrollgruppe und fehlende Verblindung. Durch die Teilnahme des operierenden Arztes an der Schulung entstanden möglicherweise Ergebnisverzerrungen aufgrund sozialer Erwünschtheit (vgl. Brosius et al., 2012). Dieser Aspekt kann insbesondere die Ergebnisse bezüglich der Beurteilung der Schulung beeinflusst haben.

Ein weiterer Verzerrungsfaktor entstand möglicherweise durch das offene Ende der Schulung. Dadurch füllten die Teilnehmenden die Fragebögen zu unterschiedlichen Zeitpunkten aus. Das unvollständige Datenmaterial kann ein Indiz dafür sein, dass die Aufmerksamkeit und Sorgfalt beim Ausfüllen der Fragebögen durch die Aufbruchsstimmung negativ beeinflusst wurde.

\section{Empfehlung für weitere Forschung}

Die Ergebnisse können die Grundlage für eine kontrollierte Studie sein, um den Nutzen und die Kosteneffektivität der Schulungsmaßnahme gegenüber Standardaufklärung $\mathrm{zu}$ untersuchen. Hier sollten dann die Fragebögen auf dem Postweg verteilt werden, um die Versuchsleiter/ inneneffekte in Richtung sozialer Erwünschtheit zu minimieren - auch wenn dies negative Einflüsse auf die Rücklaufquote und den Kostenaufwand haben könnte. Die Ein- und Ausschlusskriterien waren geeignet und sollten beibehalten werden. Um Langzeiteffekte und Einflüsse der Schulung auf die präoperative Angst nachweisen zu können, ist ein dritter Erhebungszeitpunkt kurz vor der OP anzustreben. Die Teilnehmenden sollten zudem Vorinformationen zur Notwendigkeit der postoperativen Entlastung erhalten. Eventuelle Einflussfaktoren wie Bildungsstand, Wohnsituation und Partnerschaft sollten ebenfalls untersucht werden. 


\section{FAZIT}

Die Ergebnisse dieser Studie zeigten positive Effekte der Schulungsmaßnahme in Bezug auf den Wissenszuwachs der Teilnehmenden sowie bezüglich des subjektiven Zugewinns. Die Teilnehmenden zeigten eine hohe Zufriedenheit mit dem Ablauf und den
Rahmenbedingungen der Schulung, dies bildete sich auch in den Ergebnissen der qualitativen Interviews ab. Falls langfristige Effektivität und Kosteneffizienz im Rahmen einer kontrollierten Studie gezeigt werden können, empfiehlt sich die Implementierung von präoperativen Schulungsmaßnahmen im stationären Setting.

\section{Literaturverzeichnis}

Bierhoff, H. W. (2002). Versuchsleitereffekte. In: Bierhoff, Einführung in die Sozialpsychologie. Weinheim: Beltz.

BAR (Bundesarbeitsgemeinschaft für Rehabilitation). (2005). Rehabilitation und Teilhabe.

Wegweiser für Ärzte und andere Fachkräfte der Rehabilitation. 3. Auflage. Köln: Deutscher Ärzteverlag.

Bondy, L. R., Sims, N., Schroeder, D. R., Offord, K. P., Narr, B. J. (1999). The Effect of Anesthetic

Patient Education on Preoperative Patient Anxiety. RAPM, 24 (2), $158-164$.

Bonnin, M. P., Basiglini, L., Archbold, H. A. P. (2011). What are the factors of residual pain after uncomplicated TKA? Knee Surg Sports Traumatol Arthrosc, 19 (9), 1411 - 1417.

Brosius, H. B., Haas, A. (2012). Experiment II: Validität und Fehler. In: Brosius, H. B., Haas, A., Koschel, F., Hrsg. Methoden der empirischen Kommunikationsforschung. Wiesbaden: Springer VS Verlag.

Cooil, J. \& Bithell, C. (1997). Pre-operative education for patients undergoing total hip replacement: A comparison of two methods. Physiother Theory Pract, 13, 163 - 173.

Coudeyre, E., Jardin, C., Givron, P., Ribinik, P., Revel, M., Rannou, F. (2007). Could preoperative rehabilitation modify postoperative outcomes after total hip and knee arthroplasty? Elaboration of French clinical practice guidelines. Ann Réadapt Méd Phys, 50 (3), 189 - 197.

Eyfferth, T., Bak, P., Hoffmann, R. (2011). Rehabilitation und Belastungsaufbau nach Frakturen. Trauma und Berufskrankheit, 13, 183 - 190.

Fortin, F., Kirouac, S. (1976) A randomized controlled trail of preoperative patient education. INT J NURS STUD, 13, 11 - 24.

GBE des Bundes (Gesundheitsberichterstattung des Bundes). (2012). Die 50 häufigsten Operationen der vollstationären Patientinnen und Patienten in Krankenhäusern (Rang, Anzahl, Anteil in Prozent) (Zugriff am 10.2.2015). http://www. gbebund.de/oowa921 install/servlet/oowa/aw92/dboowasys921. xwdevkit/xwd_init?gbe.isgbetol/xs_start_neu/\&p_aid=3\&p_ aid $=34913197 \&$ nummer=666\&p_sprache=D\&p_indsp=-\&p_aid= 45486167.

Giraudet-Le Quintrec, J. S., Coste, J., Vastel, L., Pacault, V., Jeanne, L., Lamas, J. P., Kerboull, L., Fougeray, M., Conseiller, C., Kahan, A., Courpied, J. P. (2003). Positive Effect of Patient Education for Hip Surgery: A Randomised Trial. CORR, 414: 112 - 120.

Heikkinen, K., Leino-Kilpi, H., Hiltunen, A., Johansson, K., Kaljonen, A., Rankinen, S., Virtanen, H., Salantera, S. (2007) Ambulatory orthopaedic surgery patients' knowledge expectations and perceptions of received knowledge. JAN 2007; 60 (3): 270 - 278.

Hintermann, B., Knupp, M., Barg, A. (2011) Mediales Schmerzsyndrom nach Sprunggelenkprothesenimplantation. Orthopäde 2011; 40: $991-999$.

Jettkowski, K., Kretschmann, J., Wurg, M. (2013). Entwicklung eines Fragebogens zur Evaluation des Wissenszuwachs, subjektiven Zugewinn und Konzept einer Patientenschulung. Unveröffentlichtes Manuskript, HAWK Hildesheim.

Johansson, K., Salanterä, S., Katajisto, J. (2007) Empowering orthopaedic patients through preadmission education: Results from a clinical study. PATIENT EDUC COUNS, 66(1), 84 - 91.

Johnson, A., Sandford, J. \& Tyndall, J. (2008) Written and verbal information versus verbal information only for patients being discharged from acute hospital settings to home. CDSR, 4.

Kaymakçı, S., Yavuz, M., Orgun, F. (2007) Surgical patient education: Turkish nursing students experiences. NURS EDUC TODAY, 27 (1), $19-25$.

McDonald, S., Hetrick, S. E., Green, S. (2008) Pre-operative education for hip or knee replacement. CDSR; 4.

McGregor, A. H., Rylands, H., Owen, A., Dore, C. J., Hughes, S. P. F. (2004) Does preoperative hip rehabilitation advice improve recovery and patient satisfaction? JOA, 19 (4), 464-468.

Niggemeyer, O., Moosig, F., Rüther, W. (2011). Operative Eingriffe an Fuß und Sprunggelenk. Rheumatol, 70, 26 - 33.

Prouty, A., Cooper, M., Thomas, P., Christensen, J., Strong, C., Bowie, L., Oermann, M. H. (2006). Multidisciplinary Patient Education for Total Joint Replacement Surgery Patients. Orthop Nurs, 25(4), $257-261$.

Ruffing, T., Muhm, M., Winkler, H. (2011). Das schmerzhafte Os intermetatarseum. Orthopäde 2011; 40: 93 - 96.

Selvan, D, Molloy, A., Mulvey, I., Abdelmalek, A., Mulvey, I. \& Alnwick, R. (2013). The effect of pre-operative foot and ankle physiotherapy group on reducing inpatient stay and improving patient care and efficiency. Foot Ankle Surg, 19(2):118-20.

Soever, L. J., MacKay, C., Saryeddine, T., Davis, A. M., Flannery, J. F., Jaglal, S. B., Levy, C., Mahomed, N. (2010). Educational Needs of Patients Undergoing Total Joint Arthroplasty. CPA, 62(3), 206 $-214$.

Zwipp, H., Grass, R. (2005). Arthrodesen des oberen Sprunggelenks nach Endoprothesenfehlschlägen. OperOrthopädTraumato, 17 (5), $18-33$. 